\title{
Allergy related outcomes at 12 months in the CORAL birth cohort of Irish children born during the first COVID 19 lockdown.
}

\author{
Sadhbh Hurley ${ }^{1}$, Ruth Franklin ${ }^{1}$, Naomi McCallion ${ }^{1}$, Aideen Byrne ${ }^{2}$, John Fitzsimons ${ }^{3}$, \\ Susan Byrne $^{1}$, Martin White ${ }^{1}$, Liam O Mahony ${ }^{4}$, and Jonathan Hourihane ${ }^{1}$ \\ ${ }^{1}$ Royal College of Surgeons in Ireland \\ ${ }^{2}$ Children's Health Ireland at Crumlin \\ ${ }^{3}$ Children's Health Ireland \\ ${ }^{4}$ University College Cork
}

January 31, 2022

Title:

Allergy related outcomes at 12 months in the CORAL Birth cohort of Irish children born during the first COVID 19 lockdown.

Word count: 1248, References: 10

Authors:

Sadhbh Hurley MB, ${ }^{1,2}$, Ruth Franklin RN ${ }^{1}$, Naomi McCallion MB,PhD ${ }^{1,3}$, Aideen M. Byrne MB, PhD ${ }^{2,4}$, John Fitzsimons MB, ${ }^{2}$, Susan Byrne MB, PhD ${ }^{1,2}$, Martin White MB, MD ${ }^{1,5}$, Liam O'Mahony PhD $^{6}$, Jonathan O'B Hourihane MB, DM ${ }^{1,2}$

1 Paediatrics and Child Health, Royal College of Surgeons in Ireland, Dublin, Ireland

2 Children's Health, Ireland, Dublin, Ireland

3 Rotunda Maternity Hospital, Dublin, Ireland

4 Trinity College, Dublin, Ireland

5 Coombe Hospital, Dublin, Ireland

6 APC Microbiome Institute, University College Cork, Cork, Ireland

Corresponding Author Information:

Jonathan O'B Hourihane

RCSI Department of Paediatrics and Child Health, Children's Health Ireland at Temple Street, Dublin 2, Ireland

Telephone: +353218784200

jonathanhourihane@rcsi.com

ORCID

Jonathan Hourihane https://orcid.org/0000-0003-4997-9857

Keywords: SARS-CoV-2; allergic outcomes; birth cohort 
Abbreviations: (alphabetical)

AD: Atopic dermatitis

ISAAC: International Study of Asthma and Allergies in Childhood (ISAAC)

LRTI: Lower Respiratory Tract Infection

SCORAD: SCORing Atopic Dermatits

SPT: Skin Prick Testing

URTI: Upper Respiratory Tract Infection

UTI: Urinary Tract Infection

Funding Source:

This study received funding from Temple Street Hospital Foundation, Dublin, Ireland, and the Clemens Von Pirquet Foundation, Geneva, Switzerland.

Conflicts of Interest:

JO'BH is a board member of Clemens Von Pirquet Foundation. No other conflicts declared.

To the Editor,

The CORAL Birth cohort is a unique birth cohort project examining allergic and autoimmune dysregulation in infants born between March and May 2020, during the first SARS-CoV-2 lockdown in Ireland. We hypothesised that the dramatic social and environmental changes imposed by the pandemic-mandated lockdown would have implications on the incidence of allergic conditions, possibly mediated by changes in microbiome diversity. We report here interim data of health outcomes, including allergic conditions, at 12 months in this cohort.

We have previously published health outcome data at 6 months. A total of 365 infants were enrolled and 344 were retained to 12 months. Allergic outcomes in the CORAL cohort were compared with a national pre-pandemic birth cohort from the BASELINE study. The 12-month appointment involved s, skin prick testing (SPT), assessment of any atopic dermatitis (AD), lateral flow SARS-CoV-2 antibody testing and stool and blood sample for analysis.

\section{Health Outcomes}

\begin{tabular}{llll}
\hline & 6 months $\mathrm{n}=354(\%)$ & 12 months $\mathrm{n}=344(\%)$ & 12 months $\mathrm{n}=344(\%)$ \\
\hline Breastfeeding & $\mathbf{1 9 2}(\mathbf{5 4})$ & $\mathbf{1 2 1}(\mathbf{3 5})$ & $\mathbf{1 2 1}(\mathbf{3 5})$ \\
Exclusive breastfeeding & $\mathbf{1 3 7}(\mathbf{3 9})$ & $\mathbf{8 1}(\mathbf{2 4})$ & $\mathbf{8 1}(\mathbf{2 4})$ \\
Vaccinations complete & $\mathbf{3 5 2}(\mathbf{9 9})$ & $\mathbf{6 7}(\mathbf{1 9 . 5})$ & $\mathbf{6 7}(\mathbf{1 9 . 5})$ \\
Vaccination scheduled & & $\mathbf{2 7 5}(\mathbf{8 0})$ & $\mathbf{2 7 5}(\mathbf{8 0})$ \\
Infant Infections & $\mathbf{9 9}(\mathbf{2 8})$ & $\mathbf{1 6 9}(\mathbf{4 9})$ & $\mathbf{1 6 9}(\mathbf{4 9})$ \\
& & Viral URTI & $131 / 169(78)$ \\
& & Otitis Media & $10 / 169(6)$ \\
& & LRTI & $9 / 169(4)$ \\
& & Gastroenteritis & $5 / 169(3)$ \\
Infant hospitalisation & $\mathbf{1 8}(\mathbf{5 . 1})$ & UTI & $4 / 169(2)$ \\
Systemic antibiotics & $\mathbf{2 2}(\mathbf{6 . 2})$ & $\mathbf{2 6}(\mathbf{7 . 6})$ & $\mathbf{2 6}(\mathbf{7 . 6})$ \\
& & $\mathbf{5 7}(\mathbf{1 7})$ & $\mathbf{5 7}(\mathbf{1 7})$ \\
Community SARS-CoV-2 & $\mathbf{0 - 6}$ months $\mathbf{n}=\mathbf{3 5 4}$ & Oral & $54 / 57(95)$ \\
PCR testing & $\mathbf{( \% )}$ & $\mathbf{6 - 1 2}$ months $\mathbf{n}=\mathbf{3 4 4}$ & $3 / 57(5)$ \\
& & $\mathbf{6 - 1 2} \mathbf{m o n t h s ~} \mathbf{n}=\mathbf{3 4 4}$ & $\mathbf{( \% )}$
\end{tabular}




\begin{tabular}{llll}
\hline & 6 months $\mathrm{n}=354(\%)$ & 12 months $\mathrm{n}=344(\%)$ & 12 months $\mathrm{n}=344(\%)$ \\
\hline & $\mathbf{2 9 ( 8 )}$ & $\mathbf{9 5}(\mathbf{2 8})$ & $\mathbf{9 5}(\mathbf{2 8})$ \\
PCR swab positivity & $\mathbf{2 / 2 9 ( 7 )}$ & $\mathbf{8 / 9 5}(\mathbf{8 . 4})$ & $\mathbf{8 / 9 5}(\mathbf{8 . 4})$ \\
\hline
\end{tabular}

Table 1: Health Outcomes at 12 months

URTI Upper Respiratory Tract infection; LRTI Lower Respiratory Tract Infection; PCR Polymerase Chain Reaction; UTI Urinary Tract Infection

Including results from both community-based PCR testing and CORAL appointment antibody testing 16/344 (4.7\%) CORAL infants had been diagnosed with SARS-2-CoV by 12 months. Despite the second and third waves prior to 12 -month appointments, antibody positivity remained low in infants at 11/344 $(3 \%)$.

Return to work was reported by $227 / 344(66 \%)$ of mothers at 12 -month review. This included mothers who returned to work from home. The infant age at return ranged from 2 to 12 months (Mean age 9.1 months). There is a statistically significant difference in breastfeeding between working and non-working mothers at 12 months $(p<0.001)$. Infants of mothers who have returned to work were more than twice as likely to be formula fed at 12 months (odds ratio 2.29; CI 95\% (1.44-3.64).

\section{Atopic Outcomes}

\section{Atopic dermatitis}

At 12 months, more CORAL infants - 87/344 (25.3\%) - had AD than in the BASELINE cohort - 232/1495 (15.5\%, p<0.0001). 74/87 CORAL infants had active AD at 12-month appointment undergoing SCORAD (SCORing AD) assessment identifying 56/74 (76\%) with mild AD (SCORAD <15), 15/74 (20\%) with moderate AD (SCORAD 15-39) and 3/74 (4\%) with severe AD (SCORAD >40).

Food introduction, sensitisation, and allergy

Milk, egg and peanut introduction, sensitisation and allergy in the CORAL group are summarised in Table 2. Sensitisation and allergy are compared between the CORAL and BASELINE cohorts at 12 months in Table 3 .

\begin{tabular}{lllll}
\hline & Milk & Egg & Egg & Peanut \\
\hline Introduced at 6 months $\mathrm{n}=354(\%)$ & $163(46)$ & $163(46)$ & $91(26)$ & $45(12)$ \\
Introduced at 12 months $\mathrm{n}=344(\%)$ & $340(99)$ & $340(99)$ & $339(98)$ & $270(78)$ \\
Sensitised at 12 months $\mathrm{n}=344(\%)$ & $4(1.2)$ & $4(1.2)$ & $20(5.8)$ & $3(0.9)$ \\
Allergic at 12 months $\mathrm{n}=344(\%)$ & $2(0.6)$ & $2(0.6)$ & $11(3.2)$ & $2(0.6)$ \\
\hline
\end{tabular}

Table 2: Food introductions, sensitisation and allergy in CORAL cohort

\begin{tabular}{lllll}
\hline & & CORAL & BASELINE & \\
\hline \multirow{3}{*}{ Any food } & Sensitisation & $\mathbf{n}=\mathbf{3 4 4}(\mathbf{\%})$ & $\mathbf{n}=\mathbf{1 5 4 0}(\mathbf{\%})$ & $\mathbf{p}$-values \\
& Allergy & $15(4.7)$ & $61(4)$ & $\mathrm{p}=0.008^{* *}$ \\
\multirow{2}{*}{ Milk } & Sensitisation & $4(1.2)$ & $22(1.4)$ & $\mathrm{p}=0.45$ \\
& Allergy & $2(0.6)$ & $19(1.2)$ & $\mathrm{p}=0.7$ \\
Egg & Sensitisation & $20(5.8) * *$ & $49(3.7)$ & $\mathrm{p}=0.3$ \\
& Allergy & $11(3.2)$ & $42(2.7)$ & $\mathrm{p}=0.02$ \\
Peanut & Sensitisation & $3(0.9)$ & $15(1)$ & $\mathrm{p}=0.9$
\end{tabular}




\begin{tabular}{llll}
\hline & CORAL & BASELINE & \\
\hline Allergy & $2(0.6)^{* *}$ & $1(0.06)$ & $\mathrm{p}=0.03^{* *}$ \\
\hline
\end{tabular}

Table 3: Food sensitisation and allergy at 12 months in CORAL and BASELINE cohorts Discussion

We hypothesised that social restrictions imposed by the SARS-CoV-2 lockdown would result in altered infant microbiome and atopic outcomes through reduced encounters with "old friend" microbes brought to the infant through family members, creche and sibling school attendance. However, changes in other related health factors, including reduced antibiotic use and increased breast feeding, which may positively affect gut microbiome diversity were also noted in this cohort.

At 12 months, less than half of CORAL babies had experienced any infective illness and just $17 \%$ had received a systemic antibiotic. 90\% of infants in a UK Birth cohort in 2008 had experienced an illness by 1 year and $80 \%$ had received a systemic antibiotic in the first year of life. The low rates of infant illness and hospitalisation in CORAL support the pre-study expectation of fewer viral infections circulating due to lockdown restrictions. Fewer antibiotics likely reflects a combination of fewer infections and less healthcare utilisation. Powerful community based epidemiological data that supports our focused prospective study has confirmed dramatically reduced admission rates for paediatric respiratory and severe bacterial infections since March 2020 when compared with the preceding 3 years.

An analysis of antibiotic consumption in 5 European countries (Austria, Italy, Germany, the Netherlands, Switzerland) identified antibiotic usage between 17- 60\% in the first year of life. Antibiotic consumption in the CORAL group at 12 months was in line with the lowest prescription rate, seen in Switzerland, in this study. Of note, the recently published EuroPrevall study demonstrated unexpectedly low prevalence of probable cow's milk and egg food allergy in Switzerland. .

Ireland has the lowest breastfeeding rate in Europe. In 2015, the National Perinatal Reporting System recorded that only $58 \%$ of babies in the Republic of Ireland were receiving any breastmilk on discharge from the maternity hospital after birth. This falls significantly to $35 \%$ of babies receiving some breastmilk at 3 months, $15 \%$ at 6 months and $11 \%$ at 9 months. In the CORAL cohort, rates were markedly different with $192 / 354(54 \%)$ of infants at 6 months and 121/344 (35\%) of infants at 12 months still receiving breast milk.

Maternal return to work has previously been associated with discontinuation of breastfeeding. In a prepandemic Irish cohort "Growing Up in Ireland", researchers identified mothers returning to part-time work were $30 \%$ more likely and those returning to full time work $113 \%$ more likely to quit breastfeeding. These differences were noted in the CORAL cohort with higher formula use at 12 months in those who had returned to work. However, $29 \%$ of mothers who had returned to work were still breastfeeding at 12 months in the CORAL group, which is dramatically higher than pre-pandemic figures. This may reflect mothers who returned to work from home and due to convenience, access or privacy were able to continue breastfeeding. This dramatic and rapid change in breastfeeding rates seen in this group of mothers may direct further research into barriers to breastfeeding in Ireland.

Despite lower antibiotic use and higher breastfeeding rates our data show AD incidence has increased in Irish infants. The AD incidence of $15.5 \%$ in the BASELINE cohort in 2008 is in keeping with incidence data from the International Study of Asthma and Allergies in Childhood (ISAAC) of between 15-20\% in 2006.

There are limited recent epidemiological data on the current incidence of $\mathrm{AD}$, especially in the first year of life. The $\mathrm{AD}$ incidence of $25.3 \%$ in the CORAL cohort may reflect a slow upward trend in $\mathrm{AD}$ since the national dataset in 2008. However, it may also reflect more recent pandemic related changes as outlined above.

Food allergen introduction continued to improve from 6 to 12 months although $20 \%$ of infants had still not yet introduced peanut at 12 months despite early weaning advice. Egg sensitisation was statistically significantly 
higher in the CORAL group which may be a result of the higher incidence of AD. This higher incidence of sensitisation has not translated into a higher incidence of egg allergy at 12 months when compared with the BASELINE cohort. This likely reflects the change in clinical practice regarding graded reintroduction of baked egg in infants with a history of reaction to egg which has led to tolerance by 12 months in most treated infants.

\section{Conclusion}

At 12 months the CORAL cohort has higher rates of egg sensitisation and $\mathrm{AD}$, but not egg allergy, when compared with a national historic dataset. Antibiotic use and infant illness and hospitalisation were much lower in this cohort and breastfeeding rates were dramatically higher than pre-pandemic Irish figures. Microbiome analysis from 6-month and 12-month appointments are ongoing and may offer further explanation for the increased sensitisation and $\mathrm{AD}$ seen in this cohort at 12 months in the context of other favourable factors including increased breastfeeding.

References

(1) Lawler M, Franklin R, McCallion N, Byrne AM, Fitzsimons J, Kinoshita M, et al. The impact of COVID19 lockdown on infants' coronavirus exposure and routine healthcare access in Ireland: The CORAL birth cohort study at 6 months. Pediatr. Allergy Immunol. 2021; 10.1111/pai.13591 [doi].

(2) Hennessy Á, Hourihane JO, Malvisi L, Irvine AD, Kenny LC, Murray DM, et al. Antenatal vitamin D exposure and childhood eczema, food allergy, asthma and allergic rhinitis at 2 and 5 years of age in the atopic disease-specific Cork BASELINE Birth Cohort Study. Allergy. 2018; 73 (11): 2182-2191. 10.1111/all.13590 [doi].

(3) Harris JM, Mills P, White C, Moffat S, Newman Taylor AJ, Cullinan P. Recorded infections and antibiotics in early life: associations with allergy in UK children and their parents. Thorax. 2007; 62 (7): 631-637. thx.2006.072124 [pii].

(4) Kadambari S, Goldacre R, Morris E, Goldacre MJ, Pollard AJ. Indirect effects of the covid-19 pandemic on childhood infection in England: population based observational study. BMJ. 2022; 376 e067519. 10.1136/bmj2021-067519.

(5) Stam J, van Stuijvenberg M, Grüber C, Mosca F, Arslanoglu S, Chirico G, et al. Antibiotic use in infants in the first year of life in five European countries. Acta Paediatrica 2012; 101 (9): 929-934. 10.1111/j.16512227.2012.02728.x [doi].

(6) Lyons SA, Clausen M, Knulst AC, Ballmer-Weber BK, Fernandez-Rivas M, Barreales L, et al. Prevalence of Food Sensitization and Food Allergy in Children Across Europe. JACI; In practice. 2020; 8 (8): 27362746.e9. S2213-2198(20)30371-8 [pii].

(7) Healthcare Pricing Office. National Perinatal Reporting System (NPRS).http://www.hpo.ie/latest_hipe_nprs_reports/NPRS_2015/Perinatal_Statistics_Report_2015. pdf [Accessed 15/12/21].

(8) Layte, R. \& McCrory, C. Maternal Health Behaviours and Child Growth in Infancy: Analyses of the Infant Cohort of the Growing Up in Ireland Study. 2014.

(9) Asher MI, Montefort S, Björkstén B, Lai CK, Strachan DP, Weiland SK, et al. Worldwide time trends in the prevalence of symptoms of asthma, allergic rhinoconjunctivitis, and eczema in childhood: ISAAC Phases One and Three repeat multicountry cross-sectional surveys. Lancet.2006; 368 (9537): 733-743. S01406736(06)69283-0 [pii].

(10) Byrne A, Kelleher M, Hourihane JO. CORRESPONDENCE BSACI 2021 guideline for the management of egg allergy. Clin Exp allergy.2021; 10.1111/cea.14061 [doi]. 